\title{
BMJ Open Protocol for the Stather Canadian Respiratory Research Outcomes Registry for Chest
ProcedurEs (SCOPE)
}

Alain Tremblay, ${ }^{1}$ Alex C Chee, ${ }^{1}$ Inderdeep Dhaliwal, ${ }^{2}$ Elaine Dumoulin, ${ }^{1}$ Ashley Gillson, ${ }^{3}$ Paul R MacEachern, ${ }^{1}$ Michael Mitchell, ${ }^{2}$ Colin Schieman, ${ }^{4}$ Daniel Stollery, ${ }^{3}$ Pen Li, ${ }^{3}$ Marc Fortin, ${ }^{5}$ Chung C Tyan, ${ }^{6}$ Erik Vakil, ${ }^{1}$ Christopher Hergott ${ }^{1}$

To cite: Tremblay A, Chee AC, Dhaliwal I, et al. Protocol for the Stather Canadian Outcomes Registry for Chest ProcedurEs (SCOPE). BMJ Open Resp Res 2021;8:e000834. doi:10.1136/ bmjresp-2020-000834

Received 13 November 2020 Revised 11 January 2021 Accepted 16 January 2021

\section{Check for updates}

(c) Author(s) (or their employer(s)) 2021. Re-use permitted under CC BY-NC. No commercial re-use. See rights and permissions. Published by BMJ.

${ }^{1}$ Medicine, University of Calgary, Calgary, Alberta, Canada

${ }^{2}$ Medicine, University of Western Ontario, London, Ontario, Canada

${ }^{3}$ Medicine, University of Alberta, Edmonton, Alberta, Canada

${ }^{4}$ Surgery, University of Calgary, Calgary, Alberta, Canada

${ }^{5}$ Respiratory Medicine, Universite Laval, Quebec, Québec, Canada

${ }^{6}$ Medicine, University of Saskatchewan College of Medicine, Saskatoon, Saskatchewan, Canada

Correspondence to Dr Alain Tremblay; atrembla@ucalgary.ca

\section{ABSTRACT}

Introduction The Stather Canadian Outcomes registry for chest ProcedurEs (SCOPE registry) is a Canadian multicentre registry of chest procedures.

Methods and analysis The SCOPE registry is designed as a multicentre prospective database of specific bronchoscopic or other pulmonary procedures. Each procedure of interest will be associated with a registry module, and data capture designed to evaluate effectiveness of procedures on relevant patient outcomes. Participating physicians will be asked to enter data for all procedures performed in a given module. The anonymised dataset will be housed in a web-based electronic secure database. Specific modules included will be based on participating physician suggestions, capacity and consensus of the steering committee and relevance of hypotheses/ research potential.

Ethics and dissemination The central registry is under approval from the Conjoint Health Research Ethics Board at the University of Calgary. We aim for registry data to lead to publication of manuscripts in international medical journals as the primary mode of dissemination. Data may also be used by local investigators for personal and/or institutional quality control purposes as well as to inform health policies. Data requests from non-participating investigators for use under ethics approved research protocols can be considered.

\section{INTRODUCTION}

Lung specialists perform a variety of medical procedures to diagnose or treat different pulmonary diseases. The scope and variety of procedures has increased dramatically over the past few years and will likely continue to expand as new technologies are developed. In general, many of these advances are technology driven, and while occasionally supported by clinical trial data the approval process for medical devices often does not require the same level of evidence as for pharmaceuticals. ${ }^{1}$ In addition, off label use of devices and procedures are common, often with little high-quality outcomes data in those settings. Further impeding the generation of highquality outcomes data is that some of the clinical applications for chest procedures occur in low volume for rare clinical indications so that single centre studies have difficulty in generating meaningful data in a timely manner. Many device and procedure studies are of retrospective design with all their methodological limitations. Finally, outcome data reported in highly controlled clinical trials with strict inclusion and exclusion criteria are not always indicative of real-world performance. ${ }^{23}$

We establish this multicentre registry of chest procedures for the diagnosis and treatment of lung diseases to increase our understanding of how these procedures impact our patients. Such procedural registries and post marketing monitoring have been advocated in the literature $^{4-6}$ and a prior bronchoscopy registry has generated important information. ${ }^{7-12}$ In the proposed project, we will prospectively collect detailed baseline and outcome data of patients undergoing a variety of lung diseaserelated diagnostic and treatment procedures at several Canadian institutions.

The project is named the Stather Canadian Outcomes registry for chest ProcedurEs registry in memory of David Stather, ${ }^{13}$ an interventional pulmonologist who had himself used procedural databases to further our understanding of complications associated with such procedures ${ }^{14-16}$ and was a pioneer in the teaching ${ }^{17-22}$ as well as development of new procedures. ${ }^{23-25}$ The project is also to be funded at its onset through the David Stather Memorial Fund. ${ }^{26}$

\section{METHODS AND ANALYSIS}

\section{Research goals}

Broad research questions and hypothesis can be generated a priori as follows, but it is fully 
expected and desired that the availability of the data collected will lead to additional opportunities for scientific investigations not foreseen at the current time. The registry will be specifically designed to collect data points allowing the following research questions:

\section{Effect of procedures on patient outcomes}

For diagnostic procedures, we seek to determine diagnostic performance characteristics of techniques in specific clinical scenarios under conditions of usual clinical practice, as well as factors affecting these measures. With regard to therapeutic procedures, technical success rates (as relevant to the procedure) as well as impact on relevant symptoms (eg, dyspnoea) or measurements (eg, pulmonary function tests) will be described under usual clinical practice conditions, as well as factors affecting these outcomes. For example, we aim to determine the success rate of lung cancer molecular diagnostic testing performed on endobronchial ultrasound samples.

\section{Monitoring for safety/complications associated with procedures}

Complication rates and factors affecting these outcomes will be investigated for each procedure type under conditions of usual clinical practice. For example, we aim to determine short term complications of bronchoscopic interventions for benign airway stenoses.

\section{Comparison of treatment outcomes between procedures for a given indication or diagnosis (Comparative Effectiveness Research)}

For a given diagnostic or therapeutic scenario, the systematic collection of outcomes data in the registry as well as

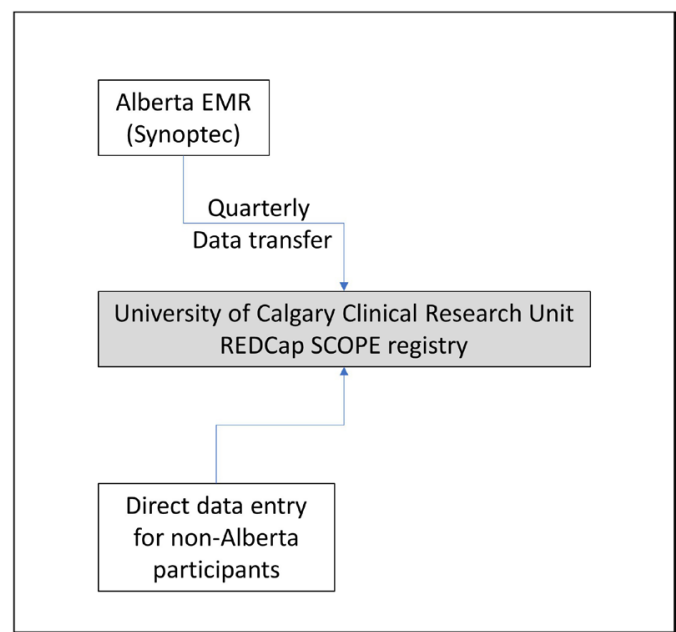

Figure 1 Structure of registry and source of data entry. Synoptec (Softworks, Edmonton) is a synoptic reporting platform in use for procedure and surgical reports within Alberta Health Services. SCOPE,Stather Canadian Outcomes registry for chest ProcedurEs. EMR: Electronic Medical Record. REDCap: Research Electronic Data Capture. baseline characteristics which may affect these outcomes can allow for different diagnostic or therapeutic procedures to be compared, using propensity matching techniques if possible. As an example, we aim to determine the sensitivity of a $19 \mathrm{G}$ transbronchial needle device in comparison to standard 21-22 G needle device for endobronchial ultrasound sampling of mediastinal lymph nodes.

\section{Quality improvement}

Between-physician and between-centre variation will be determined in terms of effectiveness as well as complication rates. Other healthcare-related factors such as trainee participation in procedures can also be tracked in the registry. Identification of variance in outcomes in different setting can then be used to develop corrective or mitigative actions, and to systematically track the impact of these actions once applied. For example, we aim to allow participants to compare their diagnostic rates for endobronchial ultrasonography in patients with sarcoidosis with that in the entire registry.

\section{Resource utilisation and policy}

Registry data on procedures can be useful to determine the utilisation and penetration of various procedures, for various indications and in different healthcare jurisdictions. Such information can be informative to healthcare administrative and policy leaders in the allocation of resources in areas of greatest need or where cost-benefit is most favourable.

\section{Registry design}

We will house the registry data in an online accessible secure database using the Research Electronic Data Capture (REDCap@) platform ${ }^{27}$ under licence from the University of Calgary Clinical Research Unit. This user friendly database allows direct real time data entry from any web-connected computer or device (eg, iPad, etc), reminders for entry of follow-up data as well as automated reporting information for individual users and data exporting functions for more detailed analytical purposes. For procedures performed within the Alberta Health Services network, the Provincial Health System in Alberta, we will extract anonymised data directly from the clinical Cancer Surgery Alberta Synoptec (Softworks, Edmonton, Canada) database established as a procedure synoptic report generation system, but which has been designed in concert with the REDCap database to capture. Physicians from other Canadian provinces will enter deidentified information directly into the REDCap portal (figure 1). As this component of the database will be deidentified, each individual physician will be required to maintain a secure patient study key linking the database ID number with their own medical record so that the can enter follow-up outcome data anonymously. The registry will not prescribe the method used to maintain 
the study key but this must meet local research ethics board approval and any other applicable privacy law and institutional policies.

We will design the database in a stepwise fashion with the development and testing of individual procedure modules in one centre before expanding access to all participants. The principal investigator's (PI) site and/ or at the site of the physician(s) who have proposed or developed the new module will perform the initial pilot testing of a new module. Individual physicians and centres can participate or not in any given module based on the procedure each performs and the resources available for data entry, but if participation is chosen they must include all procedures of that type performed for completeness and to reduce reporting bias. A log documenting each physician's participating status and dates for each module is maintained by the PI.

Any Canadian physician or surgeon actively performing procedures relevant to current registry modules and committed to reliable and timely data entry for all relevant procedures is eligible to participate. Dissemination of information about the registry has primarily occurred through discussion in the Canadian Thoracic Society Canadian Assembly for Chest Procedures Working Group.

We will apply efforts to minimise potential for biases of the registry data in the design of module and participation rules. We will consider issues surrounding generalisability, selection bias, existing user bias, confounding bias, ascertainment bias and lost to follow-up.

Query functions are built into both the Synoptec ${ }^{\mathrm{M}}$ and REDCap systems for participating physicians to easily identify procedures with outstanding outcomes data entry requirements. The PI or delegate will contact individuals with incomplete data entry at the time of quarterly data synchronisation and prior to any planned data analysis.

\section{Procedure and evaluation modules}

We will design the database to capture procedure specific/relevant data in a dynamic format where selection of a particular procedure subset results in the appropriate fields required appearing on the screen for completion. We will consider each unique procedure as a discrete event and this will form the unit of analysis. The initial roll-out of the project includes a limited number of procedure modules (figure 2), with plans to add others as participants develop experience with the process and they implement new procedures into practice.

We will define primary research hypothesis, inclusions/exclusion criteria, baseline and outcomes variables and datapoints to be collected, analysis plan and expected enrolment size/life span (stopping rules) for each module at their onset. Ideally, as we incorporate new procedures in participating centres, we will design registry modules up front to capture initial implementation, learning curves and minimise biases introduced by not including initial procedure experience for each physician.

We will consider additional modules based on participating physician suggestions, capacity and consensus of the steering committee and relevance of hypotheses/ research potential. We plan the registry as a long-term framework (indefinite) designed to evolve and change alongside the evolution of procedures in the field and associated research queries for which the registry may help clarify. We will design individual registry procedure components with anticipated finite endpoints, modifiable as new procedure variations or research questions arise from the data gathered or other developments in the field.

Investigators will submit proposals for specific analysis plans for individual research queries to the steering committee for approval prior to release of the data.

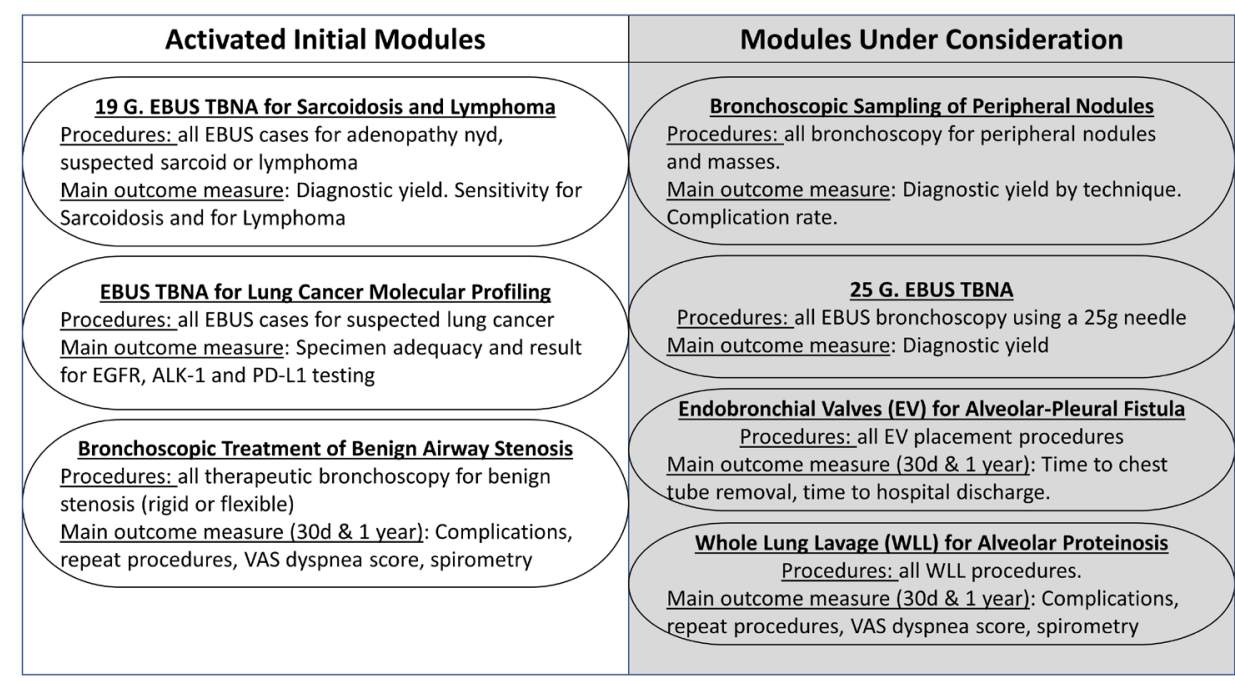

Figure 2 Initial module and under consideration. ALK-1, anaplastic lymphoma kinase-1; EBUS-TBNA, endobronchial ultrasound transbronchial needle aspiration; EGFR, epithelial growth factor receptor; ENyd, not yet diagnosed EGFR; PD-L1, programmed death-ligand; VAS, Visual Analogue Scale. 


\section{ETHICS AND DISSEMINATION}

The Conjoint Health Research Ethics Board at the University of Calgary has approved the registry (protocol REB15-2843). Each site participant(s) is responsible for local research ethics and administrative approval for the project, in particular regarding local data collection. Ideally individual physicians or sites will request a waiver of patient consent or opt-out consent procedure from each respective research ethics committee given the minimal risk of the study to participants and risk of introducing bias to the registry by not including all relevant procedures. Given the multitude of research ethics board and variations in policies and regulations across institutions and Provinces, the registry cannot prescribe a unified statement on the need for explicit consent, optout approach or waived consent.

The registry will at all times be free of identifiable patient information. Participating physicians shall maintain appropriate procedures to safeguard data confidentiality and will only supply deidentified data into the database. Individual participating physicians will retain access to their own data though the REDCap platform. In possession of only deidentified data, no possibility of data linkages to other sources will be feasible at the registry level.

The project sponsor is the University of Calgary. Registry governance will be led by a steering committee composed of the registry PI as chair and project owner accountable to the sponsor, in addition to the Chair of the Canadian Thoracic Society Canadian Assembly for Chest Procedures Working Group (or designate), one member from each actively participating centre (site PI or delegate). The committee will include a maximum of six members. Should more than five centres participate in the project, centres with the greatest number of procedures provided in the prior calendar year will be offered a position on the committee. Decision making will be by consensus, but if not achievable a majority vote will be required. The committee may establish working groups comprised of steering committee members and/ or participating physicians as needed. The chair may invite other site leads to attend meetings in a non-voting capacity at their discretion. The steering committee will aim to meet at least biannually in person or virtually. The chair will also schedule an annual general meeting open to all participating physicians and any other stakeholders identified by the steering committee.

The prospective collection of multicentre data from Canadian institutions will allow accurate assessment of diagnostic and therapeutic outcomes for this group of procedures. Such data would be expected to lead to presentation of findings at international research meetings (in abstract format) and the publication of manuscripts in international medical journals as the primary modes of dissemination. Local investigators may also use registry information for personal and/or institutional quality control purposes as well as to inform health policies that may be useful for equipment budgeting, programme funding and physician billing applications for new procedures. Non-participating investigators could also submit data requests to the steering committee for consideration for use under ethics approved research protocols. The steering committee may also consider other applications for registry data on request (scope@ucalgary.ca).

Contributors AT: Lead for registry design, concept and implementation. Prepared draft of manuscript and final submitted version. ACC: Contributed to registry design, concept, and implementation, Contributed to manuscript content and approved final version. ID: Contributed to registry implementation, Contributed to manuscript content and approved final version. ED: Contributed to registry design, concept and implementation, Contributed to manuscript content and approved final version. AG: Contributed to registry implementation, contributed to manuscript content and approved final version. PRM: Contributed to registry design, concept and implementation, contributed to manuscript content and approved final version. MM: Contributed to registry implementation, contributed to manuscript content and approved final version. CS: Contributed to registry implementation, contributed to manuscript content and approved final version. DS: Contributed to registry implementation, contributed to manuscript content and approved final version. PL: Contributed to registry implementation, contributed to manuscript content and approved final version. MF: Contributed to registry implementation, contributed to manuscript content and approved final version. CCT: Contributed to registry implementation, contributed to manuscript content and approved final version. EV: Contributed to registry implementation. Contributed to manuscript content and approved final version. $\mathrm{CH}$ : Colead for registry design, concept and implementation. Contributed to manuscript content and approved final version.

Funding This work was supported by the University of Calgary David R. Stather Memorial Fund.

Competing interests AT declares a consulting relationship with Olympus Respiratory America for work on continuing medical education programs as well as on development of bronchoscopy tools and equipment.

Patient and public involvement Patients and/or the public were not involved in the design, or conduct, or reporting, or dissemination plans of this research.

Patient consent for publication Not required.

Provenance and peer review Not commissioned; externally peer reviewed.

Open access This is an open access article distributed in accordance with the Creative Commons Attribution Non Commercial (CC BY-NC 4.0) license, which permits others to distribute, remix, adapt, build upon this work non-commercially, and license their derivative works on different terms, provided the original work is properly cited, appropriate credit is given, any changes made indicated, and the use is non-commercial. See: http://creativecommons.org/licenses/by-nc/4.0/.

\section{REFERENCES}

1 Vakil E, Tremblay A. Flying blind despite all our instruments. Chest 2020;158:1312-3.

2 Wennberg DE, Lucas FL, Birkmeyer JD, et al. Variation in carotid endarterectomy mortality in the Medicare population: trial hospitals, volume, and patient characteristics. JAMA 1998;279:1278-81.

3 Maclntyre K, Capewell S, Stewart S, et al. Evidence of improving prognosis in heart failure: trends in case fatality in 66547 patients hospitalized between 1986 and 1995. Circulation 2000;102:1126-31.

4 Pennell CP, Hirst A, Sedrakyan A, et al. Adapting the ideal framework and recommendations for medical device evaluation: a modified Delphi survey. Int J Surg 2016;28:141-8.

5 Challoner DR, Vodra WW, Devices M. Medical devices and health creating a new regulatory framework for moderate-risk devices. $N$ Engl J Med Overseas Ed 2011;365:977-9.

6 James S, Daubert J-C, Van de Werf F, Werf FVd. Commentary: use of registries to investigate the past and develop the future. BMJ 2011;342:d2826.

7 Ost DE, Ernst A, Grosu HB, et al. Complications following therapeutic bronchoscopy for malignant central airway obstruction: results of the AQuIRE registry. Chest 2015;148:450-71.

8 Ost DE, Ernst A, Grosu HB, et al. Therapeutic bronchoscopy for malignant central airway obstruction: success rates and impact on dyspnea and quality of life. Chest 2015;147:1282-98.

9 Ost DE, Ernst A, Lei X, et al. Diagnostic yield of endobronchial ultrasound-guided transbronchial needle aspiration: results of the AQuIRE bronchoscopy registry. Chest 2011;140:1557-66. 
10 Ost DE, Ernst A, Lei X, et al. Diagnostic yield and complications of bronchoscopy for peripheral lung lesions. Results of the AQuIRE registry. Am J Respir Crit Care Med 2016;193:68-77.

11 Yarmus LB, Akulian J, Lechtzin N, et al. Comparison of 21-gauge and 22-gauge aspiration needle in endobronchial ultrasound-guided transbronchial needle aspiration: results of the American College of chest physicians quality improvement registry, education, and evaluation registry. Chest 2013;143:1036-43.

12 Eapen GA, Shah AM, Lei X, et al. Complications, consequences, and practice patterns of endobronchial ultrasound-guided transbronchial needle aspiration: results of the AQulRE registry. Chest 2013;143:1044-53.

13 Tremblay A, MacEachern P, Chee A. Dr David Stather. J Bronchology Interv Pulmonol 2014;21:181.

14 Stather DR, MacEachern P, Chee A, et al. Trainee impact on procedural complications: an analysis of 967 consecutive flexible bronchoscopy procedures in an interventional pulmonology practice. Respiration 2013;85:422-8.

15 Stather DR, Maceachern P, Chee A, et al. Trainee impact on advanced diagnostic bronchoscopy: an analysis of 607 consecutive procedures in an interventional pulmonary practice. Respirology 2013;18:179-84.

16 Stather DR, MacEachern P, Chee A, et al. Safety of endobronchial ultrasound-guided transbronchial needle aspiration for patients taking clopidogrel: a report of 12 consecutive cases. Respiration 2012;83:330-4.

17 Stather DR, Chee A, Maceachern P, et al. Evaluation of a novel method of teaching endobronchial ultrasound: physician- versus respiratory therapist-proctored simulation training. Can Respir J 2013;20:243-7.
18 Stather DR, MAC EACHERN P, Chee A, et al. Evaluation of clinical endobronchial ultrasound skills following clinical versus simulation training. Respirology 2012;17:291-9.

19 Stather DR, MacEachern P, Chee A, et al. Wet laboratory versus computer simulation for learning endobronchial ultrasound: a randomized trial. Can Respir J 2012;19:325-30.

20 Stather DR, Maceachern P, Rimmer K, et al. Assessment and learning curve evaluation of endobronchial ultrasound skills following simulation and clinical training. Respirology 2011:16:698-704.

21 Stather DR, Maceachern P, Rimmer K, et al. Validation of an endobronchial ultrasound simulator: differentiating operator skill level. Respiration 2011;81:325-32.

22 Stather DR, Chee A, MacEachern P, et al. Endobronchial ultrasound learning curve in interventional pulmonary fellows. Respirology 2015;20:333-9.

23 Folch E, Santacruz JF, Fernandez-Bussy S, et al. The feasibility of EBUS-Guided TBNA through the pulmonary artery in highly selected patients. J Bronchology Interv Pulmonol 2016;23:7-13.

24 Stather DR, Tremblay A, MacEachern P, et al. Bronchoscopic removal of a large intracavitary pulmonary aspergilloma. Chest 2013;143:238-41.

25 Stather DR, Tremblay A, Dumoulin E, et al. A series of transbronchial removal of intracavitary pulmonary aspergilloma. Ann Thorac Surg 2017;103:945-50.

26 Uo C. David stather memorial fund. Available: https://netcommunity. ucalgary.ca/davidstather [Accessed 25 Sept 2020].

27 Harris PA, Taylor R, Thielke R, et al. Research electronic data capture (REDCap)--a metadata-driven methodology and workflow process for providing translational research informatics support. J Biomed Inform 2009;42:377-81. 